\title{
Contribution of binge eating behaviour to cardiometabolic risk factors in subjects starting a weight loss or maintenance programme
}

\author{
Alessandro Leone ${ }^{1 *}$, Giorgio Bedogni ${ }^{1}$, Veronica Ponissi ${ }^{1}$, Alberto Battezzati ${ }^{1}$, Valentina Beggio ${ }^{1}$, \\ Paolo Magni ${ }^{2}$, Massimiliano Ruscica ${ }^{2}$ and Simona Bertoli ${ }^{1}$ \\ ${ }^{1}$ Department of Food, Environmental and Nutritional Sciences (DeFENS), International Center for the Assessment of \\ Nutritional Status (ICANS), University of Milan, Via Sandro Botticelli 21, 20133 Milan, Italy \\ ${ }^{2}$ Department of Pharmacological and Biomolecular Sciences (DiSFeB), University of Milan, Via Balzaretti 9, 20133 Milan, Italy \\ (Submitted 14 April 2016 - Final revision received 23 September 2016 - Accepted 7 November 2016 - First published online 15 December 2016)
}

\section{Abstract}

The contribution of binge eating (BE) behaviour to cardiometabolic risk factors has been scarcely investigated so far. Previous studies have not considered the nutritional status and lifestyle of subjects suffering from BE. The aim of this study was to evaluate the contribution of BE to the metabolic syndrome (MS), its components, high total cholesterol and high LDL in a large sample of subjects, taking into account nutritional status, dietary habits, smoking status and physical activity. For this purpose, 5175 adults seeking a weight loss or maintenance programme were recruited. Anthropometrical measurements and blood parameters were measured. BE was evaluated using the Binge Eating Scale (BES). A fourteen-item questionnaire was used to evaluate the adherence to the Mediterranean diet. Smoking status and physical activity were investigated by interview. BE prevalence was 0.16 (95\% CI 0.15, 0.17). A sex- and age-adjusted Poisson regression model showed a higher prevalence of MS in binge eaters $(0 \cdot 33 ; 95 \% \mathrm{CI} 0 \cdot 28,0 \cdot 37)$ compared with non-binge eaters $(0 \cdot 27 ; 95 \% \mathrm{CI} 0 \cdot 25,0 \cdot 28, P=0 \cdot 011)$. However, the statistical difference was lost after inclusion of BMI and lifestyle parameters in the multiple-adjusted model. We also evaluated the association between the continuous outcomes of interest and the BES score using a multivariable median regression model. We observed a positive, but clinically irrelevant, association between BES score and HDL levels $(P<0 \cdot 001)$. In conclusion, BE does not seem to be independently related to cardiometabolic risk factors. However, the screening and treatment of BE are of clinical relevance in order to reduce the risk of developing obesity.

\section{Key words: Binge eating: Cross-sectional studies: Epidemiology: Lifestyle: Metabolic syndrome}

Binge eating (BE) is a behaviour characterised by eating in a discrete period of time an amount of food that is definitely larger than what most people would eat in a similar period of time under similar circumstances, accompanied by a sense of lack of control over eating. According to the Diagnostic and Statistical Manual of Mental Disorders, 5th edition ${ }^{(1)}$, when such behaviour occurs at least once weekly for 3 months without compensatory behaviours, it is defined as binge eating disorder (BED). BE is also common in other eating disorders such as bulimia nervosa (BN), some subtypes of anorexia nervosa (AN) and eating disorders not otherwise specified $(\mathrm{EDNOS})^{(1)}$. It is particularly prevalent in subjects seeking a weight loss or maintenance programme, among whom BE prevalence is $17 \%$, with increased prevalence in women as well as in younger and in obese subjects ${ }^{(2)}$. Beyond the higher risk of developing obesity, binge eaters have a worse dietary pattern $^{(3)}$, a higher smoking status ${ }^{(4)}$ and lower physical activity levels than subjects free of $\mathrm{BE}^{(2)}$.
Obesity and lifestyle are crucial factors associated with cardiometabolic diseases $^{(5,6)}$, whereas the contribution of $\mathrm{BE}$ behaviour is still a debated topic ${ }^{(7)}$. A 5-year longitudinal study of 134 individuals with BED compared with age- and sex-matched controls (the authors tried to match even for BMI, but failed in their purpose) showed an increased risk for dyslipidemia in subjects with BED, after adjusting for sex, age, baseline BMI and BMI changes. The authors concluded that BED might confer a risk of components of the metabolic syndrome (MS) over and above the risk attributable to obesity alone $^{(8)}$. Subsequently, a cross-sectional study carried out on 3551 participants of the Framingham Heart Study reported that subjects with objective $\mathrm{BE}$, but not subjects with subclinical $\mathrm{BE}$, had a higher risk of impaired fasting glucose compared with non-binge eaters, after adjusting for sex, age, BMI, smoking status, alcohol intake, education and depressive symptoms ${ }^{(9)}$. A further cross-sectional study carried out on 2225 patients waiting for bariatric surgery reported a positive association

Abbreviations: BE, binge eating; BED, binge eating disorder; BES, Binge Eating Scale; Medscore, Mediterranean score; MRM, median regression model; MS, metabolic syndrome; PWRM, Poisson working regression model.

* Corresponding author: Dr A. Leone, fax +3902 50216077, email alessandro.leone1@unimi.it 
between BED status and impaired fasting glucose, after controlling for age, sex, education, BMI and psychiatric disorders $^{(10)}$. More recently, a 16-year longitudinal study observed a higher incidence of type 2 diabetes mellitus in subjects with BED and BN, but not AN, compared with age- and sex-matched controls ${ }^{(11)}$. All these evidences suggest that BE may increase the likelihood of developing components of $\mathrm{MS}^{(7)}$. Nevertheless, whether this increased risk is attributable to an independent contribution of $\mathrm{BE}$ or to a higher presence of obesity and unhealthy lifestyle is unclear ${ }^{(7)}$. Previous studies, indeed, have not taken, or only partially taken, into account the effects of nutritional status and lifestyle on cardiometabolic risk factors. Therefore, the relative contribution of $\mathrm{BE}$ to cardiometabolic risk is presently unknown.

In this context, the aim of this study was to evaluate the contribution of BE to cardiometabolic risk in a large sample of subjects starting a weight loss or maintenance programme, taking into account the potential effects of nutritional status, dietary habits, smoking status and physical activity.

\section{Methods}

\section{Study design}

We performed a cross-sectional study on 5466 consecutive adults who self-referred to the International Center for the Assessment of Nutritional Status (University of Milan) from September 2010 to January 2015, in order to participate in a structured weight loss or weight maintenance programme. On the same day, they underwent a clinical examination, an anthropometric assessment and a structured interview by a trained dietitian, in order to obtain information about marital status, educational level, smoking status and structured physical activity. Physical activity was investigated by asking the subjects the following questions: 'Do you practice any structured physical activity?' and 'How many hours per week do you spend on this activity?'. Subjects who spent $\geq 2 \mathrm{~h} /$ week performing any structured physical activity were considered as active $^{(2)}$. Moreover, all the patients filled in two questionnaires to evaluate adherence to the Mediterranean diet ${ }^{(12,13)}$ and the presence of $\mathrm{BE}^{(14,15)}$. From the initial number of subjects recruited for the study, thirty subjects were excluded because they were diagnosed as having acute infective, neurological, gastrointestinal, cardiac, renal and pulmonary disorders or were unable to understand and fill in the questionnaires. The present study was carried out according to the Declaration of Helsinki, and all participants gave their written informed consent to participate. The institutional review board approved the study procedures.

\section{Anthropometric measurements}

Anthropometric measurements were obtained according to conventional criteria and measuring procedures proposed by Lohmann et al. ${ }^{(16)}$. Body weight (BW, kg) was measured using Column scale (Seca 700 balance; Seca Corporation) up to $100 \mathrm{~g}$ with subjects wearing only light underwear and after bladder emptying. Body height $(\mathrm{BH}, \mathrm{cm})$ was measured to the nearest
$0 \cdot 1 \mathrm{~cm}$ using a vertical stadiometer. BMI was calculated using the following formula: BMI $\left(\mathrm{kg} / \mathrm{m}^{2}\right)=\mathrm{BW}(\mathrm{kg}) / \mathrm{BH}^{2}\left(\mathrm{~m}^{2}\right)$. BMI was classified into four categories: underweight (BMI $<18.5 \mathrm{~kg} / \mathrm{m}^{2}$ ), normal weight (BMI $18.5-24.9 \mathrm{~kg} / \mathrm{m}^{2}$ ), overweight (BMI $25 \cdot 0-29.9 \mathrm{~kg} / \mathrm{m}^{2}$ ) and obese $\left(\right.$ BMI $>30.0 \mathrm{~kg} / \mathrm{m}^{2}$ ). Waist circumference was measured midway between the lower rib margin and the superior anterior iliac spine to the nearest $0.5 \mathrm{~cm}$ with a non-stretch tape applied horizontally.

\section{Laboratory measurements}

Fasting total cholesterol, HDL-cholesterol, LDL-cholesterol, TAG and glucose were measured using enzymatic methods (Cobas Integra 400 Plus; Roche Diagnostics) ${ }^{(17)}$. Blood pressure was measured by a physician using a random-zero mercury sphygmomanometer following the Joint National Committee 7 guidelines ${ }^{(18)}$.

\section{The metabolic syndrome and clinical outcomes}

The MS was diagnosed using the harmonised international definition $^{(19)}$. Large waist was defined as waist circumference $\geq 102 \mathrm{~cm}$ in men and $\geq 88 \mathrm{~cm}$ in women, low HDL-cholesterol as values $<40 \mathrm{mg} / \mathrm{dl}$ in men and $<50 \mathrm{mg} / \mathrm{dl}$ in women, high TAG as values $\geq 150 \mathrm{mg} / \mathrm{dl}$ or treatment with TAG-lowering drugs, high blood pressure as systolic blood pressure $\geq 130 \mathrm{mmHg}$ or diastolic blood pressure $\geq 85 \mathrm{mmHg}$ or treatment with pressurelowering drugs, and high glucose as values $\geq 100 \mathrm{mg} / \mathrm{dl}$ or treatment with glucose-lowering drugs. The MS was defined as three or more of the above components. In addition, high total cholesterol was defined as total cholesterol $\geq 200 \mathrm{mg} / \mathrm{dl}$ and high $\mathrm{LDL}$ as values $\geq 130 \mathrm{mg} / \mathrm{dl}$.

\section{Mediterranean dietary pattern}

Adherence to the Mediterranean diet was evaluated using a validated fourteen-item questionnaire ${ }^{(13)}$, which is the extension of an original nine-item questionnaire ${ }^{(12)}$. A fourteenitem Mediterranean score (Medscore) was obtained from this questionnaire following the guidelines of the PREvención con DIeta MEDiterránea (PREDIMED) study (www.predimed.es) and a specific validation study by Schröder et al. ${ }^{(13)}$ with some adaptations already used in previous studies ${ }^{(3,20-22)}$. In brief, 1 point was attributed for each of the following: (1) olive oil as the main cooking fat, (2) olive oil $\geq 4$ tablespoons/d, (3) vegetables $\geq 2$ servings/d ( $\geq 1$ portion raw or on salad), (4) fruits $\geq 3$ servings/d, (5) red or processed meat $<1$ serving/d, (6) butter or cream or margarine $<1 / \mathrm{d}$, (7) soda drinks $<1 / \mathrm{d}$, (8) wine $\geq 3$ glasses/week, (9) legumes $\geq 3$ servings/week, (10) fish/seafood $\geq 3$ servings/week, (11) commercial sweets and confectionery $<3 /$ week, (12) nuts $\geq 1$ /week, (13) white more than red meats (yes) and (14) use of sofrito sauce $\geq 2$ /week. Subjects with a MEDscore $\geq 9$ points were considered to have a high adherence to the Mediterranean $\operatorname{diet}^{(3,21,22)}$.

\section{Psychological assessment}

Eating behaviour was evaluated using the Italian version of the Binge Eating Scale (BES) ${ }^{(14,15)}$. BES consists of sixteen 
forced-choice questions, each with a set of three to four answer choices. BES gives a score ranging from 0 to 46. BE was defined as a BES score $\geq 18^{(23)}$. The questionnaire was considered invalid when more than $10 \%$ of items was missing. Following such criteria, further 261 subjects were excluded.

\section{Statistical analysis}

Statistical analyses were performed on a final number of 5175 subjects. Most continuous variables had non-Gaussian distributions, and all are reported as 25th, 50th and 75th percentiles. Discrete variables are reported as counts and frequencies.

A Poisson working regression model (PWRM) with robust $95 \%$ CI was used to estimate prevalence and its predictors ${ }^{(24,25)}$. A PWRM was used because a binomial regression model failed to converge for some of the regressions of interest. Expectedly, the estimates made by the binomial regression model and by the PWRM were similar in all cases where both converged. Sex- and age-adjusted and multiple-adjusted PWRM were used to evaluate the associations of MS, its components, high cholesterol and high LDL with BES. The response variable of all PWRM (MS, MS components, high cholesterol, low LDL) was discrete $(0=$ no; $1=$ yes). Besides BES (discrete, $0=$ non-binge eater; $1=$ binge eater, or continuous, score units), predictors of the multivariable PWRM were the following: (1) sex (discrete, $0=$ female; $1=$ male), (2) age (continuous, years/10), (3) BMI (continuous, $\mathrm{kg} / \mathrm{m}^{2}$ ), (4) Medscore (continuous, score units), (5) smoking status (discrete, $0=$ no; $1=\mathrm{ex} ; 2=$ yes) and (6) physical activity (discrete, $0=$ no; $1=$ yes). Adjusted probabilities of the responses of interest were calculated from the PWRM ${ }^{(26)}$.

We also evaluated the association between the continuous MS components (TAG, HDL, glucose, systolic blood pressure and diastolic blood pressure), total cholesterol and LDLcholesterol and the BES score using a multivariable median regression model (MRM) using the same covariates of the PWRM - that is, sex, age, BMI, Medscore, smoking status and physical activity. Adjusted estimates of the responses of interest were calculated from the $\mathrm{MRM}^{(26)}$.

Degree 2 multivariable fractional polynomials (MFP) were used to test whether the relationships of continuous predictors with the responses were non-linear ${ }^{(27)}$. MFP selected an inverse square root transformation of BMI for all PWRM models and no transformation, a $\log _{e}$ transformation, an inverse transformation or an inverse square-root transformation for age depending on the model. There was only a modest gain in the linearity of continuous predictors when MFP were applied to the MRM; therefore, all continuous covariates were kept untransformed for the MRM analysis. Adjusted estimates of the responses of interest were calculated from the $\mathrm{MRM}^{(26)}$.

Statistical analyses were performed using Stata 14.2 (Stata Corporation LP).

\section{Results}

The continuous measurements of the 5175 study subjects are given in Table 1 . Women made up $72 \%$ of the study population.
The distribution of age, BMI status, BE, MS and lifestyle factors is given in Table 2 .

The prevalence of $\mathrm{BE}$ in the pooled sample was 0.16 (95\% CI $0 \cdot 15,0 \cdot 17)$ and was higher in women $(0 \cdot 20 ; 95 \%$ CI $0 \cdot 19,0 \cdot 21)$ than in men (0.07; 95\% CI 0.06, 0.08, $P<0 \cdot 001$, PWRM).

The prevalence and prevalence rate ratios (PRR) of MS, its components, high total cholesterol and high LDL-cholesterol are given in Table 3 .

The prevalence of MS in the pooled sample was 0.28 (95\% CI $0 \cdot 26,0 \cdot 29)$. This prevalence was higher in binge eaters $(0.33$; $95 \%$ CI $0.28,0 \cdot 37)$ compared with non-binge eaters $(0.27 ; 95 \%$ CI $0 \cdot 25,0 \cdot 28)$ in the sex- and age-adjusted model $(P=0 \cdot 011$, PWRM). However, the statistical difference was lost after inclusion of nutritional status and lifestyle factors in the multiple-adjusted model. The prevalence of high blood pressure was marginally higher in binge eaters $(0.52 ; 95 \% \mathrm{CI}$ $0.47,0.57)$ compared with non-binge eaters $(0.47 ; 95 \%$ CI 0.45 , $0 \cdot 49)$ in the sex- and age-adjusted model ( $P=0 \cdot 076$, PWRM). However, the statistical difference was lost after inclusion of nutritional status and lifestyle factors in the multiple-adjusted model. No differences in the prevalence of high TAG, low HDL, high glucose, high total cholesterol and high LDL were observed between binge eaters and non-binge eaters in both models.

Table 4 reports the PRR of MS, its components, high total cholesterol and high LDL associated with an increase of 1 unit in the BES score in the sex- and age-adjusted model and in the multiple-adjusted model (sex, age, BMI, Medscore, smoking status and physical activity).

We observed an increased risk in MS, high blood pressure and high glucose associated with an increase of 1 unit in the BES score in the sex- and age-adjusted model. However, such increments were no more significant in the multiple-adjusted model. Only a weak inverse association was observed between the BES score and the risk of low HDL.

Fig. 1 plots the prevalence of MS, its components, high cholesterol and high LDL as a function of continuous BES, taking into account the effects of sex, age, BMI, Medscore, smoking status and physical activity (PWRM, see the 'Statistical analysis' section for details). The values graphed on the $x$-axis represent the 5 th, 25th, 50th, 75th and 95th percentiles of the BES score.

Fig. 2 plots the median values of TAG, total cholesterol, HDL, LDL, glucose, systolic blood pressure and diastolic blood pressure as a function of continuous BES, taking into account the effects of sex, age, BMI, Medscore, smoking status and physical activity (MRM, see the 'Statistical analysis' section for details). The values graphed on the $x$-axis represent the 5 th, 25th, 50th, 75th and 95th percentiles of the BES score. The association with BES was linear in all cases and was statistically significant only for HDL, which was higher in patients with BE $(P<0 \cdot 001)$

\section{Discussion}

This is the first study to explore, in a large sample, the association between $\mathrm{BE}$ and cardiometabolic risk factors, taking into account the effects of nutritional status and lifestyle. 
Table 1. Measurements of the study subjects

\begin{tabular}{|c|c|c|c|c|c|c|c|c|c|}
\hline & \multicolumn{3}{|c|}{ Women (n 3719) } & \multicolumn{3}{|c|}{ Men $(n$ 1456) } & \multicolumn{3}{|c|}{ Total $(n 5175)$} \\
\hline & $P_{50}$ & $\mathrm{P}_{25}$ & $\mathrm{P}_{75}$ & $P_{50}$ & $\mathrm{P}_{25}$ & $\mathrm{P}_{75}$ & $P_{50}$ & $\mathrm{P}_{25}$ & $\mathrm{P}_{75}$ \\
\hline Age (years) & 46 & 37 & 54 & 47 & 39 & 56 & 46 & 38 & 55 \\
\hline Weight $(\mathrm{kg})$ & 73.1 & 65.4 & 83.0 & 91.5 & 82.8 & $102 \cdot 1$ & 78.0 & 68.0 & $90 \cdot 1$ \\
\hline Height (m) & 1.62 & 1.58 & 1.66 & 1.75 & 1.71 & 1.79 & 1.65 & 1.60 & 1.71 \\
\hline BMI $\left(\mathrm{kg} / \mathrm{m}^{2}\right)$ & 27.9 & 25.0 & 31.6 & $30 \cdot 1$ & $27 \cdot 3$ & 33.1 & 28.5 & $25 \cdot 6$ & 32.2 \\
\hline Waist circumference $(\mathrm{cm})$ & $92 \cdot 0$ & 84.0 & $100 \cdot 6$ & 105.5 & 97.6 & 114.0 & 95.8 & $86 \cdot 7$ & 105.4 \\
\hline TAG (mg/dl) & 85 & 63 & 116 & 116 & 84 & 162 & 92 & 68 & 129 \\
\hline Total cholesterol $(\mathrm{mg} / \mathrm{dl})$ & 210 & 185 & 237 & 210 & 185 & 238 & 210 & 185 & 237 \\
\hline HDL-cholesterol (mg/dl) & 62 & 54 & 73 & 48 & 41 & 56 & 58 & 49 & 69 \\
\hline LDL-cholesterol (mg/dl) & 129 & 107 & 152 & 138 & 115 & 161 & 131 & 109 & 155 \\
\hline Glucose (mg/dl) & 91 & 86 & 98 & 98 & 91 & 105 & 93 & 87 & 101 \\
\hline Systolic blood pressure (mmHg) & 120 & 110 & 130 & 130 & 120 & 140 & 120 & 110 & 130 \\
\hline Diastolic blood pressure $(\mathrm{mmHg})$ & 80 & 70 & 80 & 80 & 80 & 85 & 80 & 70 & 80 \\
\hline BES & 10 & 5 & 16 & 7 & 4 & 11 & 9 & 5 & 15 \\
\hline Medscore & 7 & 6 & 8 & 7 & 5 & 8 & 7 & 6 & 8 \\
\hline
\end{tabular}

$P_{50}$, 50th percentile; $P_{25}, 25$ th percentile; $P_{75}, 75$ th percentile; BES, Binge Eating Scale score; Medscore, Mediterranean diet score.

Our study confirms that $\mathrm{BE}$ is common in patients seeking a weight loss or maintenance programme, with higher prevalence in women than in men ${ }^{(2)}$. Moreover, we found an increased risk of MS, high blood pressure and high glucose with increasing BES scores in the sex- and age-adjusted model. However, such associations were lost after inclusion of nutritional status and lifestyle factors in the model. Only an inverse, but clinically irrelevant, association was found between BES score and low HDL risk. These results are of great relevance in order to better understand the independent contribution of BE to cardiometabolic risk. The present study, indeed, adds to the information that $\mathrm{BE}$ is unlikely related to MS and other metabolic parameters. On the basis of such findings, and on previous evidences suggesting an association between BE and obesity, low adherence to the Mediterranean diet ${ }^{(3)}$, a higher smoking status $^{(4)}$ and low physical activity level ${ }^{(2)}$, we can speculate that BE leads to MS through the development of obesity and of an unhealthy lifestyle. However, only a longitudinal trial can confirm such a speculation.

Previous case-control studies, carried out on small samples of obese patients with BED, have not found any significant difference in binge eating episodes or severity of eating disorder psychopathology between subjects with and without $\mathrm{MS}^{(28,29)}$. Nevertheless, Roehrig et $a l^{(28)}$ found that some lifestyle behaviours including fewer episodes of weight cycling and regular meal skipping were significant predictors of MS. However, Blomquist et $a l .{ }^{(29)}$ failed to replicate these findings, adding that dietary restraint, depressive symptoms or self-esteem did not differ between subjects with and without MS. A non-significant association between BE and MS has also been reported in two cross-sectional studies ${ }^{(9,10)}$ and in a longitudinal study ${ }^{(8)}$. In agreement with these studies, we did not find any increased risk of MS with increasing BE severity after inclusion of nutritional status and lifestyle factors.

Even though the two cross-sectional studies involving subjects of the Framingham Heart Study ${ }^{(9)}$ and patients waiting for bariatric surgery ${ }^{(10)}$ did not find any association between BE and MS, they observed a higher risk of impaired fasting glucose in subjects with BED compared with subjects without BED, after adjustment for nutritional status and other potential confounders. However, they did not adjust for important lifestyle factors such as dietary pattern and physical activity, and this could be the reason for the discrepancy between the results. In fact, we observed that the initial significant association between BES score and the risk of impaired fasting glucose was lost after adjusting for nutritional status and lifestyle factors. These considerations may also explain why our findings are inconsistent with results reported by Raevuori et al. ${ }^{(11)}$ and de Jonge et $\mathrm{ll}^{\left({ }^{(30)}\right.}$. These two studies, using, respectively, a longitudinal ${ }^{(11)}$ and cross-sectional design ${ }^{(30)}$, observed an increased risk of type 2 diabetes mellitus in subjects with BED and BN. However, BMI and lifestyle data were not available, and therefore all analyses were not adjusted for nutritional status and other confounders. Moreover, as the authors stated, subjects with BED were more prone to develop overweight and obesity $^{(11)}$. These strong limitations do not allow to establish whether the increased risk of diabetes is attributable to BE or to a worse nutritional status.

In contrast, a longitudinal study, carried out on a small sample of overweight and obese individuals, reported a higher 5-year incidence of dyslipidemia in subjects with BED compared with age- and sex-matched controls after adjustment for nutritional status ${ }^{(8)}$. Even though this study is worthy for its longitudinal design, it has the strong limitation of using selfreported metabolic values, which may be less accurate, and thus the quantification of associations would have been less reliable. Moreover, they did not take into account any variable describing the lifestyle of individuals, and, for example, a lower physical activity level and a higher sedentary lifestyle, which are behaviours typical in subjects with $\mathrm{BED}^{(31)}$, may be the reasons for the higher risk of dyslipidemia. Our findings show an increment in serum HDL levels with increasing BES scores. However, the difference was small and clinically irrelevant. In fact, it should be noted that the difference in the serum level of HDL-cholesterol between BES scores corresponding to the 5 th and 95th percentiles was only approximately $3.5 \mathrm{mg} / \mathrm{dl}$. 
Table 2. Distribution of age, BMI status, Binge Eating Scale (BES), lifestyle factors and the metabolic syndrome (MS) (Numbers and percentages)

\begin{tabular}{|c|c|c|c|c|c|c|}
\hline & \multicolumn{2}{|c|}{ Women } & \multicolumn{2}{|c|}{ Men } & \multicolumn{2}{|c|}{ Total } \\
\hline & $n$ & $\%$ & $n$ & $\%$ & $n$ & $\%$ \\
\hline \multicolumn{7}{|l|}{ Age (decade) } \\
\hline 18-19 years & 30 & 0.8 & 5 & 0.3 & 35 & 0.7 \\
\hline $20-29$ years & 349 & $9 \cdot 4$ & 89 & $6 \cdot 1$ & 438 & 8.5 \\
\hline 30-39 years & 798 & 21.5 & 298 & $20 \cdot 5$ & 1096 & $21 \cdot 2$ \\
\hline 40-49 years & 1139 & $30 \cdot 6$ & 472 & 32.4 & 1611 & $31 \cdot 1$ \\
\hline 50-59 years & 871 & $23 \cdot 4$ & 331 & $22 \cdot 7$ & 1202 & $23 \cdot 2$ \\
\hline $60-69$ years & 448 & 12 & 210 & $14 \cdot 4$ & 658 & $12 \cdot 7$ \\
\hline$\geq 70$ years & 84 & $2 \cdot 3$ & 51 & 3.5 & 135 & 2.6 \\
\hline \multicolumn{7}{|l|}{ BMI class } \\
\hline Normal weight (BMI 18.5-24.9 kg/m²) & 930 & 25 & 130 & $8 \cdot 9$ & 1060 & $20 \cdot 5$ \\
\hline Overweight (BMI $\left.25 \cdot 0-29.9 \mathrm{~kg} / \mathrm{m}^{2}\right)$ & 1515 & $40 \cdot 7$ & 589 & $40 \cdot 5$ & 2104 & $40 \cdot 7$ \\
\hline Obesity class $1\left(\right.$ BMI $\left.30.0-34.9 \mathrm{~kg} / \mathrm{m}^{2}\right)$ & 861 & $23 \cdot 2$ & 523 & 35.9 & 1384 & $26 \cdot 7$ \\
\hline Obesity class 2 (BMI $\left.35.0-39.9 \mathrm{~kg} / \mathrm{m}^{2}\right)$ & 318 & $8 \cdot 6$ & 181 & $12 \cdot 4$ & 499 & $9 \cdot 6$ \\
\hline Obesity class $3\left(\mathrm{BMI} \geq 40.0 \mathrm{~kg} / \mathrm{m}^{2}\right)$ & 95 & $2 \cdot 6$ & 33 & $2 \cdot 3$ & 128 & 2.5 \\
\hline \multicolumn{7}{|l|}{ Binge eating $(B E S \geq 18)$} \\
\hline No & 2977 & 80 & 1353 & 92.9 & 4330 & 83.7 \\
\hline Yes & 742 & 20 & 103 & $7 \cdot 1$ & 845 & $16 \cdot 3$ \\
\hline \multicolumn{7}{|l|}{ Physical activity } \\
\hline No & 2214 & $59 \cdot 5$ & 780 & $53 \cdot 6$ & 2994 & 57.9 \\
\hline Yes & 1505 & $40 \cdot 5$ & 676 & $46 \cdot 4$ & 2181 & $42 \cdot 1$ \\
\hline \multicolumn{7}{|l|}{ Smoker } \\
\hline No & 2054 & $55 \cdot 2$ & 678 & $46 \cdot 6$ & 2732 & $52 \cdot 8$ \\
\hline Yes & 776 & 20.9 & 280 & $19 \cdot 2$ & 1056 & $20 \cdot 4$ \\
\hline Ex & 889 & 23.9 & 498 & $34 \cdot 2$ & 1387 & $26 \cdot 8$ \\
\hline \multicolumn{7}{|l|}{ Medscore } \\
\hline 3 & 77 & $2 \cdot 1$ & 40 & $2 \cdot 7$ & 117 & $2 \cdot 3$ \\
\hline 4 & 272 & $7 \cdot 3$ & 111 & $7 \cdot 6$ & 383 & $7 \cdot 4$ \\
\hline 5 & 550 & $14 \cdot 8$ & 222 & $15 \cdot 2$ & 772 & 14.9 \\
\hline 6 & 809 & $21 \cdot 8$ & 318 & $21 \cdot 8$ & 1127 & 21.8 \\
\hline 7 & 912 & 24.5 & 307 & $21 \cdot 1$ & 1219 & 23.6 \\
\hline 8 & 608 & $16 \cdot 3$ & 250 & $17 \cdot 2$ & 858 & $16 \cdot 6$ \\
\hline 9 & 328 & 8.8 & 138 & 9.5 & 466 & 9 \\
\hline 10 & 123 & $3 \cdot 3$ & 54 & 3.7 & 177 & 3.4 \\
\hline 11 & 40 & $1 \cdot 1$ & 16 & $1 \cdot 1$ & 56 & $1 \cdot 1$ \\
\hline \multicolumn{7}{|c|}{ Adherence to the Mediterranean diet (Medscore $\geq 9$ ) } \\
\hline No & 3228 & $86 \cdot 8$ & 1248 & $85 \cdot 7$ & 4476 & $86 \cdot 5$ \\
\hline Yes & 491 & $13 \cdot 2$ & 208 & $14 \cdot 3$ & 699 & $13 \cdot 5$ \\
\hline \multicolumn{7}{|c|}{ High waist circumference ( $\geq 102 \mathrm{~cm}$ for men; $\geq 88 \mathrm{~cm}$ for women) } \\
\hline No & 1389 & $37 \cdot 3$ & 549 & $37 \cdot 7$ & 1938 & 37.4 \\
\hline Yes & 2330 & $62 \cdot 7$ & 907 & $62 \cdot 3$ & 3237 & $62 \cdot 6$ \\
\hline High TAG $(\geq 150 \mathrm{mg} / \mathrm{dl}$ or TAG-lowering $\mathrm{dr}$ & & & & & & \\
\hline No & 3254 & 87.5 & 1002 & $68 \cdot 8$ & 4256 & $82 \cdot 2$ \\
\hline Yes & 465 & $12 \cdot 5$ & 454 & $31 \cdot 2$ & 919 & $17 \cdot 8$ \\
\hline Low HDL $(<40 \mathrm{mg} / \mathrm{dl}$ in $\mathrm{men} ;<50 \mathrm{mg} / \mathrm{dl}$ in & & & & & & \\
\hline No & 3124 & 84 & 1181 & $81 \cdot 1$ & 4305 & $83 \cdot 2$ \\
\hline Yes & 595 & 16 & 275 & $18 \cdot 9$ & 870 & $16 \cdot 8$ \\
\hline High blood pressure $(\geq 130 / 85 \mathrm{mmHg}$ or $p$ & ering $d r$ & & & & & \\
\hline No & 2269 & 61 & 437 & 30 & 2706 & $52 \cdot 3$ \\
\hline Yes & 1450 & 39 & 1019 & 70 & 2469 & $47 \cdot 7$ \\
\hline High glucose $(\geq 100 \mathrm{mg} / \mathrm{dl}$ or glucose-lowe & & & & & & \\
\hline No & 2910 & $78 \cdot 2$ & 821 & $56 \cdot 4$ & 3731 & $72 \cdot 1$ \\
\hline Yes & 809 & $21 \cdot 8$ & 635 & $43 \cdot 6$ & 1444 & $27 \cdot 9$ \\
\hline MS score & & & & & & \\
\hline 0 & 890 & 23.9 & 157 & $10 \cdot 8$ & 1047 & $20 \cdot 2$ \\
\hline 1 & 1075 & 28.9 & 275 & $18 \cdot 9$ & 1350 & $26 \cdot 1$ \\
\hline 2 & 965 & 25.9 & 379 & 26 & 1344 & 26 \\
\hline 3 & 564 & $15 \cdot 2$ & 385 & $26 \cdot 4$ & 949 & $18 \cdot 3$ \\
\hline 4 & 173 & $4 \cdot 7$ & 198 & $13 \cdot 6$ & 371 & $7 \cdot 2$ \\
\hline 5 & 52 & 1.4 & 62 & $4 \cdot 3$ & 114 & $2 \cdot 2$ \\
\hline MS ( $\geq 3$ components) & & & & & & \\
\hline No & 2930 & $78 \cdot 8$ & 811 & $55 \cdot 7$ & 3741 & $72 \cdot 3$ \\
\hline Yes & 789 & $21 \cdot 2$ & 645 & $44 \cdot 3$ & 1434 & $27 \cdot 7$ \\
\hline High cholesterol ( $\geq 200 \mathrm{mg} / \mathrm{dl})$ & & & & & & \\
\hline No & 1467 & 39.4 & 601 & $41 \cdot 3$ & 2068 & $40 \cdot 0$ \\
\hline Yes & 2252 & $60 \cdot 6$ & 855 & $58 \cdot 7$ & 3107 & $60 \cdot 0$ \\
\hline High LDL-cholesterol ( $\geq 130$ mg/dl) & & & & & & \\
\hline No & 1950 & $52 \cdot 4$ & 629 & $43 \cdot 2$ & 2579 & $49 \cdot 8$ \\
\hline Yes & 1769 & $47 \cdot 6$ & 827 & $56 \cdot 8$ & 2596 & $50 \cdot 2$ \\
\hline
\end{tabular}


Table 3. Prevalence and prevalence rate ratios (PRR) of the metabolic syndrome (MS), MS components, high cholesterol and high LDL in binge eaters and non-binge eaters $\dagger$

(Prevalence, PRR and robust $95 \%$ confidence intervals obtained from Poisson working regression model using BES score as discrete variables (BES < 18: non-binge eaters, BES $\geq 18$ : binge eaters)

\begin{tabular}{|c|c|c|c|c|c|c|c|c|c|c|c|c|}
\hline & \multicolumn{6}{|c|}{ Sex- and age-adjusted model } & \multicolumn{6}{|c|}{ Multiple-adjusted model } \\
\hline & \multicolumn{2}{|c|}{ Binge eaters } & \multicolumn{2}{|c|}{ Non-binge eaters } & \multirow[b]{2}{*}{ PRR } & \multirow[b]{2}{*}{$95 \% \mathrm{Cl}$} & \multicolumn{2}{|c|}{ Binge eaters } & \multicolumn{2}{|c|}{ Non-binge eaters } & \multirow[b]{2}{*}{ PRR } & \multirow[b]{2}{*}{$95 \% \mathrm{Cl}$} \\
\hline & Prevalence & $95 \% \mathrm{Cl}$ & Prevalence & $95 \% \mathrm{Cl}$ & & & Prevalence & $95 \% \mathrm{Cl}$ & Prevalence & $95 \% \mathrm{Cl}$ & & \\
\hline MS & 0.33 & $0.28,0.37$ & 0.27 & $0.25,0.28$ & $1 \cdot 21^{* *}$ & $1.04,1.40$ & 0.26 & $0.22,0.29$ & 0.28 & $0.26,0.30$ & 0.92 & $0.80,1.07$ \\
\hline High TAG & 0.20 & $0.17,0.24$ & 0.17 & $0.16,0.19$ & $1 \cdot 15$ & $0.96,1.39$ & 0.18 & $0.15,0.21$ & 0.18 & $0.17,0.19$ & 1.00 & $0.83,1.21$ \\
\hline Low HDL & 0.18 & $0.15,0.21$ & 0.17 & $0.15,0.18$ & 1.09 & $0.91,1.30$ & 0.16 & $0.13,0.18$ & 0.17 & $0.16,0.18$ & 0.92 & $0.77,1.10$ \\
\hline High blood pressure & 0.52 & $0.47,0.57$ & 0.47 & $0.45,0.49$ & 1.11 & $0.99,1.24$ & 0.47 & $0.42,0.52$ & 0.48 & $0.46,0.50$ & 0.97 & $0.87,1.09$ \\
\hline High glucose & 0.31 & $0.27,0.35$ & 0.27 & $0.26,0.29$ & $1 \cdot 12$ & $0.96,1.30$ & 0.27 & $0.24,0.31$ & 0.28 & $0.26,0.30$ & 0.98 & $0.84,1.14$ \\
\hline High total cholesterol & 0.61 & $0.55,0.66$ & 0.60 & $0.58,0.62$ & 1.02 & $0.92,1.12$ & 0.60 & $0.55,0.66$ & 0.60 & $0.58,0.62$ & 1.01 & $0.91,1.11$ \\
\hline High LDL & 0.52 & $0.47,0.57$ & 0.50 & $0.48,0.52$ & 1.04 & $0.93,1.16$ & 0.50 & $0.45,0.55$ & 0.50 & $0.48,0.52$ & 1.00 & $0.90,1 \cdot 12$ \\
\hline
\end{tabular}

BES, Binge Eating Scale; Medscore, Mediterranean score

${ }_{* \star}^{*} P<0.01$

† The multiple-adjusted model included sex, age, BMI, Medscore, smoking status and physical activity as covariates.

Table 4. Prevalence rate ratios (PRR) of the metabolic syndrome (MS), MS components, high cholesterol and high LDL associated with an increase of 1 unit in Binge Eating Scale (BES) before and after correction for confounders (sex, age, BMI, Medscore, smoking status and physical activity) $\dagger$

(PRR and robust $95 \%$ confidence intervals obtained from Poisson working regression model using the BES score as continuous variable)

\begin{tabular}{|c|c|c|c|c|c|c|c|c|c|c|c|c|c|c|}
\hline & \multicolumn{2}{|c|}{ MS } & \multicolumn{2}{|c|}{ High TAG } & \multicolumn{2}{|c|}{ Low HDL } & \multicolumn{2}{|c|}{ High blood pressure } & \multicolumn{2}{|c|}{ High glucose } & \multicolumn{2}{|c|}{ High total cholesterol } & \multicolumn{2}{|c|}{ High LDL } \\
\hline & PRR & $95 \% \mathrm{Cl}$ & PRR & $95 \% \mathrm{Cl}$ & PRR & $95 \% \mathrm{Cl}$ & PRR & $95 \% \mathrm{Cl}$ & PRR & $95 \% \mathrm{Cl}$ & PRR & $95 \% \mathrm{Cl}$ & PRR & $95 \% \mathrm{Cl}$ \\
\hline Sex-and & $1.02^{* \star *}$ & $1.01,1.02$ & 1.01 & $0.99,1.02$ & 1.00 & $0.99,1.01$ & $1 \cdot 01^{\star \star}$ & $1.00,1.01$ & $1.01^{\star \star \star}$ & $1.01,1.02$ & 1.00 & $1.00,1.00$ & 1.00 & $1.00,1.00$ \\
\hline Multiple-adjusted model & 0.99 & $0.99,1.00$ & 0.99 & $0.98,1.00$ & $0.99^{\star \star}$ & $0.98,1.00$ & 1.00 & $0.99,1.00$ & 1.00 & $0.99,1.01$ & 1.00 & $1.00,1.00$ & 1.00 & $0.99,1.01$ \\
\hline
\end{tabular}

Medscore, Mediterranean score.

${ }^{\star *} P<0.01,{ }^{* \star *} P<0.001$

$\dagger$ The multiple-adjusted model included sex, age, BMI, Medscore, smoking status and physical activity as covariates. 

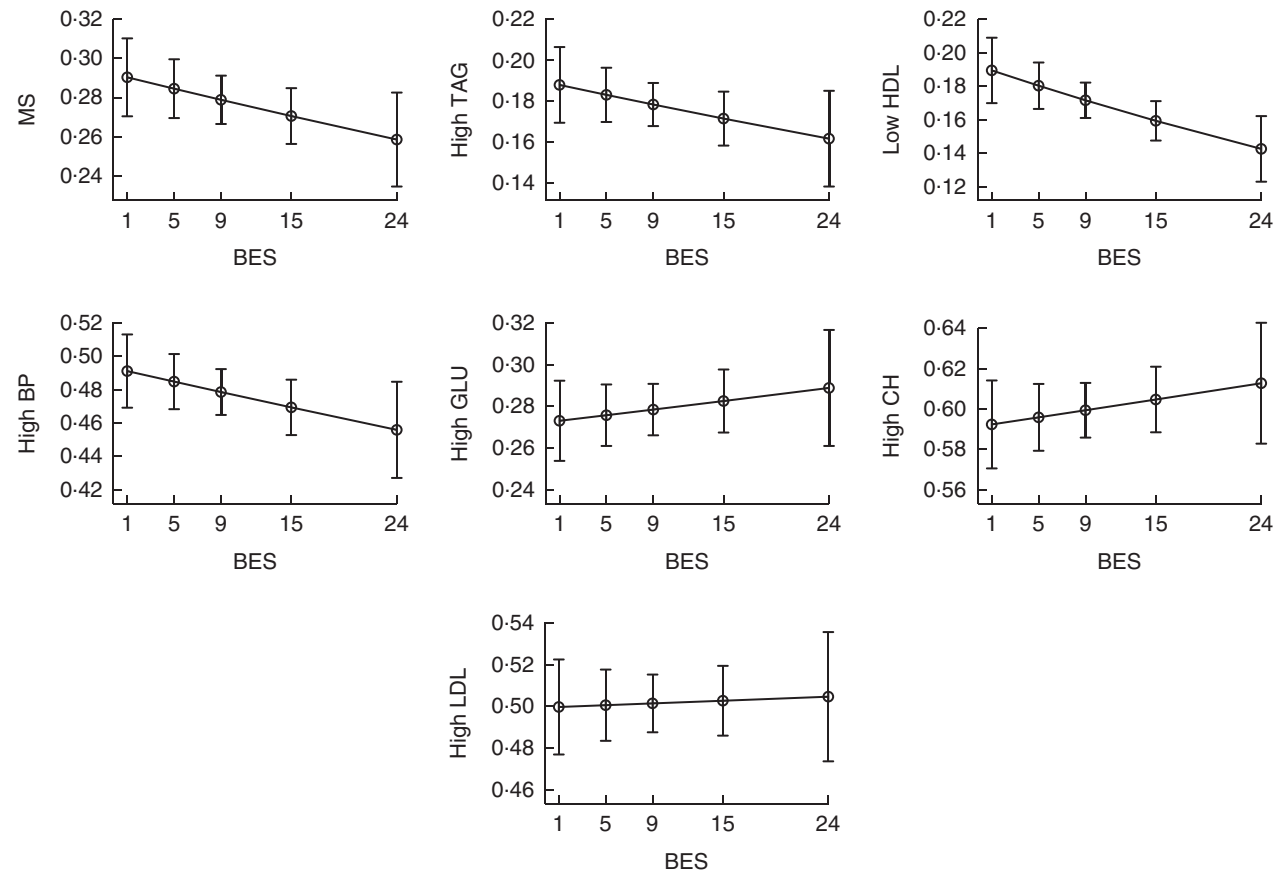

Fig. 1. Prevalence of the metabolic syndrome (MS), components of the MS, high cholesterol and high LDL as a function of continuous Binge Eating Scale (BES). Values are adjusted probabilities estimated from the Poisson working regression model described under the 'Statistical analysis' section. The values graphed on the $x$-axis represent the 5 th, 25 th, 50 th, 75 th and 95 th percentiles of BES. Only the relationship with low HDL was statistically significant $(P=0.007)$. BP, blood pressure; $\mathrm{GLU}$, glucose; $\mathrm{CH}$, total cholesterol.
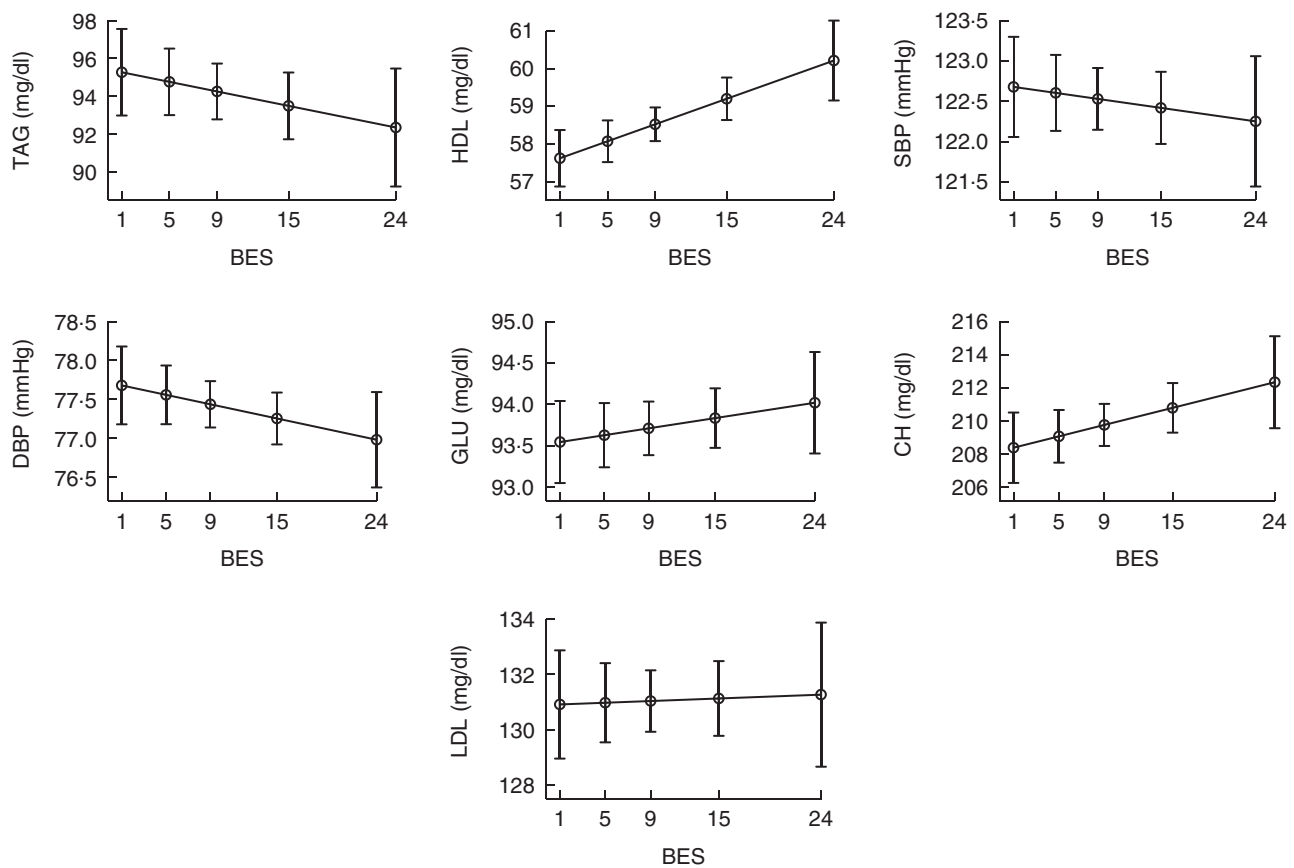

Fig. 2. Values of laboratory measurements as a function of continuous Binge Eating Scale (BES). Values are adjusted probabilities estimated from the median regression model described under the 'Statistical analysis' section. The values graphed on the $x$-axis represent the 5th, 25th, 50th, 75th and 95th percentiles of BES. Only the relationship with $\mathrm{HDL}$ level was statistically significant $(P<0.001)$. MS, metabolic syndrome; SBP, systolic blood pressure; DBP, diastolic blood pressure; GLU, glucose; $\mathrm{CH}$, total cholesterol.

Strengths of this study include its sample size and the fact that we are the first to adjust the analysis for nutritional status and lifestyle variables such as adherence to the Mediterranean diet, smoking status and physical activity. Moreover, we decided to include normal weight subjects as well, as we recently showed that this category of individuals, especially young 
women, is not BE-free ${ }^{(2)}$. However, it is not free of limitations. First, its cross-sectional nature does not allow us to establish a cause-effect relationship. Second, we enrolled subjects seeking a weight loss or maintenance programme, and therefore our results cannot be extrapolated to the general population. Third, we did not have any information concerning the onset of binging. Finally, we focused on BE, the primary diagnostic criterion for several disorders including BED, BN, AN and EDNOS, whose presence, however, does not necessarily involve the presence of an objective eating disorder.

In conclusion, $\mathrm{BE}$ does not seem to confer a risk of MS and other metabolic parameters over and above the risk attributable to obesity and lifestyle alone. However, considering the higher risk for developing obesity and having an unhealthy lifestyle among subjects suffering from BE, the screening and treatment of $\mathrm{BE}$ are issues of clinical relevance for the prevention of obesity and cardiometabolic diseases.

\section{Acknowledgements}

The authors thank the ICANS research staff and especially Chiara Caporali, Giulia De Carlo, Valentina Giustizieri, Chiara Lessa, Lidia Lewandowski, Diana Osio and Giovanna Croce for their help during this study.

This study was supported by ICANS internal grants.

A. L. and S. B. designed the research; A. L., V. P., A. B., V. B., P. M., M. R. and S. B. conducted the research; A. L. and G. B. performed statistical analysis; A. L. wrote the manuscript; A. L. had primary responsibility for the final content; and all authors read and approved the final manuscript.

The authors have no conflicts of interest to declare.

\section{References}

1. American Psychiatric Association (2013) Diagnostic and Statistical Manual of Mental Disorders, 5th ed. Arlington, VA: American Psychiatric Publishing.

2. Bertoli S, Leone A, Ponissi V, et al. (2016) Prevalence of and risk factors for binge eating behaviour in 6930 adults starting a weight loss or maintenance programme. Public Health Nutr 19, 71-77.

3. Bertoli S, Spadafranca A, Bes-Rastrollo M, et al. (2015) Adherence to the Mediterranean diet is inversely related to binge eating disorder in patients seeking a weight loss program. Clin Nutr 34, 107-114.

4. Solmi M, Veronese N, Sergi G, et al. (2016) The association between smoking prevalence and eating disorders: a systematic review and meta-analysis. Addiction 111, 1914-1922.

5. Sperling LS, Mechanick JI, Neeland IJ, et al. (2015) The cardiometabolic health alliance: working toward a new care model for the metabolic syndrome. J Am Coll Cardiol 66 , 1050-1067.

6. Gomez-Huelgas R, Jansen-Chaparro S, Baca-Osorio AJ, et al. (2015) Effects of a long-term lifestyle intervention program with Mediterranean diet and exercise for the management of patients with metabolic syndrome in a primary care setting. Eur J Intern Med 26, 317-323.

7. Mitchell JE (2016) Medical comorbidity and medical complications associated with binge-eating disorder. Int J Eat Disord 49, 319-323.
8. Hudson JI, Lalonde JK, Coit CE, et al. (2010) Longitudinal study of the diagnosis of components of the metabolic syndrome in individuals with binge-eating disorder. Am J Clin Nutr 91, 1568-1573.

9. Abraham TM, Massaro JM, Hoffmann U, et al. (2014) Metabolic characterization of adults with binge eating in the general population: the Framingham Heart Study. Obesity (Silver Spring) 22, 2441-2449.

10. Mitchell JE, King WC, Pories W, et al. (2015) Binge eating disorder and medical comorbidities in bariatric surgery candidates. Int J Eat Disord 48, 471-476.

11. Raevuori A, Suokas J, Haukka J, et al. (2015) Highly increased risk of type 2 diabetes in patients with binge eating disorder and bulimia nervosa. Int J Eat Disord 48, 555-562.

12. Martinez-Gonzalez MA, Fernandez-Jarne E, Serrano-Martinez M, et al. (2004) Development of a short dietary intake questionnaire for the quantitative estimation of adherence to a cardioprotective Mediterranean diet. Eur J Clin Nutr 58, 1550-1552.

13. Schröder H, Fito M, Estruch R, et al. (2011) A short screener is valid for assessing Mediterranean diet adherence among older Spanish men and women. J Nutr 141, 1140-1145.

14. Gormally J, Black S, Daston S, et al. (1982) The assessment of binge eating severity among obese persons. Addict Behav $\mathbf{7}$, $47-55$.

15. Di Bernardo M, Barciulli E, Ricca V, et al. (1998) Validazione della versione italiana della Binge Eating Scale in pazienti obesi (Binge Eating Scale in obese patients: validation of the Italian version). Minerva Psichiatr 39, 125-130.

16. Lohmann TG, Roche AF \& Martorell R (1988) Antbropometric Standardization Reference Manual. Champaign, IL: Human Kinetics Books.

17. Spadafranca A, Cappelletti C, Leone A, et al. (2015) Relationship between thyroid hormones, resting energy expenditure and cardiometabolic risk factors in euthyroid subjects. Clin Nutr 34, 674-678.

18. Chobanian AV, Bakris GL, Black HR, et al. (2003) Seventh report of the Joint National Committee on Prevention, Detection, Evaluation, and Treatment of High Blood Pressure. Hypertension 42, 1206-1252.

19. Alberti KG, Eckel RH, Grundy SM, et al. (2009) Harmonizing the metabolic syndrome: a joint interim statement of the International Diabetes Federation Task Force on Epidemiology and Prevention; National Heart, Lung, and Blood Institute; American Heart Association; World Heart Federation; International Atherosclerosis Society; and International Association for the Study of Obesity. Circulation 120, 1640-1645.

20. Babio N, Bulló M, Basora J, et al. (2009) Adherence to the Mediterranean diet and risk of metabolic syndrome and its components. Nutr Metab Cardiovasc Dis 19, 563-570.

21. Bertoli S, Leone A, Vignati L, et al. (2015) Adherence to the Mediterranean diet is inversely associated with visceral abdominal tissue in Caucasian subjects. Clin Nutr 34, 1266-1272.

22. Soldati L, Bertoli S, Terranegra A, et al. (2014) Relevance of Mediterranean diet and glucose metabolism for nephrolithiasis in obese subjects. J Transl Med 12, 34 .

23. Marcus MD, Wing RR \& Hopkins J (1988) Obese binge eaters: affect, cognitions, and response to behavioural weight control. J Consult Clin Psychol 56, 433-439.

24. Lumley T, Kronmal R \& Ma S (2006) Relative risk regression in medical research: models, contrasts, estimators, and algorithms. UW Biostatistics Working Paper Series, Working Paper 293. Seattle: University of Washington.

25. Barros AJ \& Hirakata VN (2003) Alternatives for logistic regression in cross-sectional studies: an empirical comparison of models that directly estimate the prevalence ratio. $B M C$ Med Res Methodol 3, 21. 
26. Williams R (2012) Using the margins command to estimate and interpret adjusted predictions and marginal effects. Stata J 12, 308-331.

27. Royston P \& Sauerbrei W (2008) Multivariable Model-Building: A Pragmatic Approach to Regression Analysis Based on Fractional Polynomials for Modelling Continuous Variables. Chichester: John Wiley \& Sons.

28. Roehrig M, Masheb RM, White MA, et al. (2009) The metabolic syndrome and behavioral correlates in obese patients with binge eating disorder. Obesity (Silver Spring) 17, 481-486.
29. Blomquist KK, Milsom VA, Barnes RD, et al. (2012) Metabolic syndrome in obese men and women with binge eating disorder: developmental trajectories of eating and weight-related behaviors. Compr Psychiatry 53, 1021-1027.

30. de Jonge P, Alonso J, Stein DJ, et al. (2014) Associations between DSM-IV mental disorders and diabetes mellitus: a role for impulse control disorders and depression. Diabetologia 57, 699-709.

31. Levine MD, Marcus MD \& Moulton P (1996) Exercise in the treatment of binge eating disorder. Int J Eat Disord 19, 171-177. 